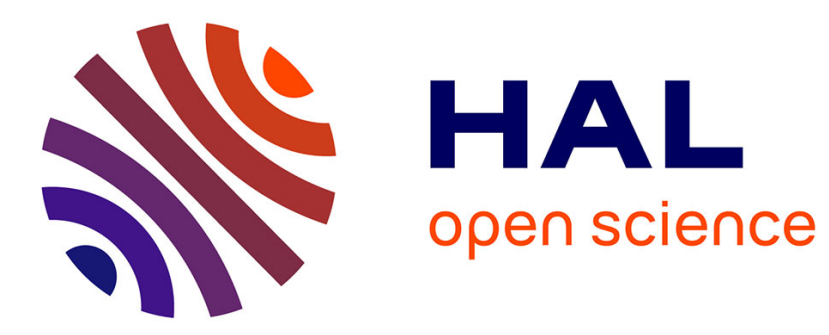

\title{
Le style déductif du Conseil d'État et la ligne de partage des mots
}

Pierre Brunet

\section{To cite this version:}

Pierre Brunet. Le style déductif du Conseil d'État et la ligne de partage des mots. Droit et Société, 2015, pp.545 - 561. 10.3917/drs.091.0545 . halshs-01416513

\section{HAL Id: halshs-01416513 https://shs.hal.science/halshs-01416513}

Submitted on 14 Dec 2016

HAL is a multi-disciplinary open access archive for the deposit and dissemination of scientific research documents, whether they are published or not. The documents may come from teaching and research institutions in France or abroad, or from public or private research centers.
L'archive ouverte pluridisciplinaire HAL, est destinée au dépôt et à la diffusion de documents scientifiques de niveau recherche, publiés ou non, émanant des établissements d'enseignement et de recherche français ou étrangers, des laboratoires publics ou privés. 


\section{Droit \& Société, 2015/3 (N 91), p. 545-561 \\ Disponible sur Cairn}

\section{Le style déductif du Conseil d'État et la ligne de partage des mots}

\section{Pierre Brunet}

\section{Professeur à l'Université Paris 1 Panthéon Sorbonne}

Le style des décisions du Conseil d'État est encore aujourd'hui de l'ordre du «formel " et semble réservé à une élite : les décisions s'adressent en fait essentiellement aux juristes dont certains sont eux-mêmes juges, nationaux ou supranationaux. C'est d'ailleurs pour mieux se faire comprendre de ces autres juges que le Conseil d'État réfléchit à une modification de son style rédactionnel. Pour autant, il n'est nullement question d'abandonner l'idéal de la motivation déductive et concise pensée sur le modèle rigoureux du syllogisme. Cette concision est compensée par les conclusions des rapporteurs qui, elles, fournissent les raisons officieuses des décisions officielles.

The style of the judgments from the Conseil d'Etat is still today very formal and seems to be reserved for an elite : judgments are less addressed to plaintiffs than to other jurists whom many are in fact national or supranational judges. It is in order to be best understood by them that the Conseil d'Etat is considering a change in the writing style of public law judgments. Though, there is no discussion about the ideal of deduction and concision in the motivation which still modeled as a rigorous syllogism. Such a concision is tempered by the conclusions of the rapporteurs which, them, can contain the informal reasons of the formal decisions.

À l'évidence, le terme qui, depuis l'origine, revient sur toutes les lèvres pour qualifier le style du Conseil d'État est celui de "laconisme». II a d'ailleurs inspiré des jugements très ambivalents ou du moins nuancés, certains allant jusqu'à y voir une forme de mutisme ${ }^{1}$. II s'exprime sous deux formes bien connues: une motivation minimale des décisions, elle-même enfermée dans un syllogisme aussi concis

\footnotetext{
J'ai tiré un très grand profit des discussions que j'ai eues avec de nombreux collègues qui ont accepté de relire ce texte. Je tiens à les en remercier chaleureusement.

${ }^{1}$ André DE LAUBADĖRE, "Le Conseil d'État et l'incommunicabilité », Études et documents du Conseil d'État, (31), 1979-1980, p. 17, ici p. 19: « Le souci de concision (...) qui relève de l'exercice de style lui vaut un respect que laisse apparaître la qualification d'imperatoria brevitas mais qui n'en oppose pas moins un certain obstacle à une compréhension facile, complète et assurée de la signification des décisions (...) Le laconisme rend ainsi parfois difficile l'accès à certains aspects de la jurisprudence. Le mutisme le rend impossible ».
} 
qu'impeccable, et une rédaction adoptant une phrase unique par laquelle le Conseil d’État déroule ce syllogisme.

Vu sous cet angle, le style du Conseil d'État ressemble à une caricature de Formal Style que Llewellyn dépeignait comme une «idéologie orthodoxe » ${ }^{2}$ selon laquelle les juges ne doivent créer aucune règle, aucun principe, aucune théorie propre et se limiter à appliquer les règles selon un principe de division du travail clair et simple : au législateur la décision des choix de valeurs et de société, aux juges l'application des règles créées par le législateur. Au Formal Style, Llewellyn opposait le Grand Style, là le juge ne se borne pas à l'application de la lettre de la règle : le droit que produit le Grand Style incorpore les raisons des règles elles-mêmes, et le sens commun de la cour. Le Grand Style est, comme l'écrit Twining, le « style des raisons articulées $\|^{3}$.

À s'en tenir là, on court toutefois le risque de réduire le Conseil d'État au cliché qui voit les décisions des cours françaises engoncées dans un formalisme précieux et creux $^{4}$. Or, la jurisprudence de la juridiction administrative n'est pas détachable des travaux annexes et connexes que sont les conclusions des rapporteurs publics (hier, les commissaires du gouvernement) ${ }^{5}$. Les analyses proposées par Mitchel Lasser à propos de la Cour de Cassation ont mis en évidence que cette dernière ne se complaît pas dans les froideurs du formalisme et qu'elle a aussi recours à des

\footnotetext{
2 Karl N. LleWellyn, The Common Law Tradition-Deciding Appeals, Boston: Little, Brown and Company, 1960, p. 37-38: "The Formal Style is of peculiar interest to us because it set the picture against which all modern thinking has played - call it, as of the last eighty or ninety years, "the orthodox ideology". That picture is clean and clear : the rules of law are to decide the cases ; policy is for the legislature, not for the courts, and so is change even in pure common law. "

${ }^{3}$ William TWINING, Karl Llewellyn and the Realist Movement, London : Weidenfeld and Nicholson, 1973, p. 228.

${ }^{4}$ Hein KöTZ, "The Role of the Judge in the Court-Room: The Common Law and Civil Law Compared », Journal of South African Law (1), 1987, p. 35, ici p. 41, à propos de la Cour de cassation : "The style of French judicial opinions (...) are generally marked by polished elegance and formal clarity, but they also seem to be frozen in a pedantic ritual of empty formalism, inappropriate to the uniqueness of the concrete facts of life behind the case which can often only be guessed at. "

${ }^{5}$ On pourrait, pour bien faire, ajouter également la chronique de l'AJDA. Sur celle-ci, v. Xavier DOMINO, "La chronique de jurisprudence à I'AJDA. Le point de vue du chroniqueur ", in Jacques Caillosse et Olivier Renaudie, Le Conseil d'État et l'Université, Paris : Dalloz, coll. "Thèmes et commentaire », 2015, p. 147 et Olivier RENAUDIE, " La chronique de jurisprudence de l'AJDA. Le point de vue de l'Universitaire », ibid., p. 153.
} 
arguments de politique jurisprudentielle ${ }^{6}$. Si la démonstration est convaincante pour la Cour de cassation, elle l'est plus encore pour le Conseil d'État.

Enfin, celui-ci n'est pas complètement indifférent à l'idée d'exercer une influence ; sa jurisprudence récente témoigne d'une recherche active des moyens de participer à l'évolution du droit européen afin que les décisions des cours nationales soient prises en compte. Cette quête d'influence n'est pas non plus étrangère à la réflexion menée au sein du Conseil d'État sur le style rédactionnel de ses décisions. Le rapport rendu par le Groupe de travail sur la rédaction des décisions de la juridiction administrative présidé par Philippe Martin en avril 2012 envisage diverses réformes tendant à simplifier la rédaction tout en conservant le style concis et efficace de la motivation .

On voudrait ici s'arrêter sur la relation délicate et complexe entre le style rédactionnel et le style de la motivation. La remise en cause de l'un risque-t-elle ou a-t-elle des chances (c'est selon...) d'avoir des conséquences sur l'autre ? II y a quelques raisons d'en douter car, à coté des décisions rendues, le Conseil d'État ${ }^{9}$ dispose de cet outil que sont les conclusions des rapporteurs publics pour articuler les raisons profondes de ses décisions. On s'intéressera donc à trois éléments distinctifs et constitutifs du style du Conseil d'État : le mode de rédaction, la motivation officielle de ses décisions et leur motivation officieuse dans les conclusions.

\section{Le style rédactionnel : une évolution nécessaire, sans révolution prévisible}

\footnotetext{
${ }^{6}$ Mitchel LASSER, « Judicial (Self-)Portraits : Judicial Discourse in the French Legal System », Yale Law Journal, 104 (6), 1995, p. 1325 ; ID., Judicial Deliberations. A Comparative Analysis of Judicial Transparency and Legitimacy, Oxford : Oxford University Press, 2004.

${ }^{7}$ On pense, évidemment, à la solution Arcelor de 2007, v. infra.

${ }^{8}$ GROUPE DE TRAVAIL SUR LA RÉDACTION DES DÉCISIONS DE LA JURIDICTION ADMINISTRATIVE, avril 2012, (cité infra Rapport Martin). Son contenu est aisément disponible: http://www.conseiletat.fr/content/download/1690/5098/version/1/file/rapport_redaction_decisions_juradm_2012.pdf. II a déjà fait l'objet de plusieurs excellents commentaires : v. Christophe FARDET, "Le style des décisions du Conseil d'État » Revue d'Histoire des Facultés de Droit et de la Science Juridique, (33), 2013, p. 219 et Jacques PETIT, "La motivation des décisions du juge administratif », in Sylvie CAUDAL (dir.), La motivation en droit public, Paris : Dalloz, 2013, p. 213 voir aussi la réaction de Gilles Pellissier dans le même volume et encore Gilles PELLISSIER, "La réflexion de la juridiction administrative sur la rédaction de ses décisions ", Les Cahiers de la Justice, 6 (2), 2014, p. 219.

9 L'espace limité de cet article nous contraint à parler par synecdoque du Conseil d'État pour la juridiction administrative tout entière.
} 
Le style du Conseil d'État, c'est d'abord, à l'évidence, une certaine façon d'écrire ${ }^{10}$. La doctrine qui s'intéresse au style dans lequel sont rédigées les motivations de la juridiction administrative souligne, depuis plusieurs années, une évolution qui témoignerait d'un effort de "lisibilité » ou du moins de "pédagogie ». Ce dernier s'expliquerait par le changement dans l'office du juge administratif lequel assumerait désormais " un office de législateur et un office d'administrateur »; la décision du juge serait devenue un « acte d'explication »; on serait passé « de l'énigme à la pédagogie ${ }^{11}$. Au point que l'on peut considérer que la motivation est utilisée comme « un instrument de régulation permettant au juge à la fois de maîtriser les effets d'une décision rendue, mais aussi d'agir sur la fabrication et l'application du droit administratif au seul cas d'espèce ॥".

L'explication de ce changement de rédaction - cette lisibilité plus grande - par un autre changement - le juge se faisant administrateur - n'est cependant pas dépourvue de circularité dans la mesure où l'on ne peut saisir le changement d'office qu'à travers la pédagogie dont ferait preuve un juge qui désirerait être lisible. En réalité, si changement il y a, il est ailleurs et bien davantage dans les nouveaux pouvoirs de contrôle des actes de l'administration dont le juge administratif dispose aujourd'hui, parce qu'il s'en est doté ou parce que le droit national ou celui européen les lui a reconnus. Or, la mise en œuvre de ces pouvoirs est de plus en plus complexe et les juges susceptibles de les utiliser eux-mêmes plus nombreux. La pédagogie entend donc moins répondre à une exigence de lisibilité qu'à une exigence d'unification de l'action des juridictions administratives, ou encore, et pour le dire d'un mot, de cohérence du droit produit par les juridictions administratives. Autrement dit, en faisant preuve de pédagogie, le Conseil d'État exerce sa fonction de juge de cassation de façon préventive. II n'est pas l'administrateur qu'il fut hier mais le manager ${ }^{13}$ qu'appelle notre époque.

\footnotetext{
${ }^{10}$ Laquelle fait l'objet de normes internes peu accessibles aux profanes. Malgré mes efforts, je n'ai pas eu accès à la circulaire NORMA ni au « Guide du rapporteur ».

${ }^{11}$ Olivier LE BOT, « Rédaction des jugements et lisibilité des décisions des juges administratifs », in Michel PAILLET (dir.), La modernisation de la justice administrative en France, Bruxelles: Larcier, 2010, p. 281-296, p. 288 : « il en résulte la recherche d'une plus grande lisibilité dans la rédaction de ses décisions ॥. II fait remarquer que le Tribunal administratif de Lyon a pris l'initiative de changements dans la présentation de ses propres jugements.

12 Virginie DONIER, «Style et structure des décisions du Conseil d'État: vers une évolution culturelle? », in Fabrice HOURQUEBIE et Marie-Claire PONTHOREAU (dir.), op. cit., p. 197, ici p. 199.

${ }^{13}$ Hein KÖTZ, art. cit., p. 42
} 
Or, l'expérience prouve que la phrase unique - qui n'empêche pourtant pas certains obiter dicta ${ }^{14}$ - ne parvient plus à remplir la fonction qui était hier la sienne - faire ressortir l'évidence par la brièveté ou, comme le disait ses partisans, tracer un "chemin rectiligne tout entier orienté vers le dispositif »" Elle apparaît non pas désuète mais parfaitement inadéquate dès lors que la juridiction administrative s'en sert pour y faire tenir plusieurs discours destinés autant aux juridictions subordonnées qu'aux juridictions européennes. C'est notamment ce qui arrive lorsque le Conseil d'État pose une règle, générale certes, mais faite de plusieurs plis ; une règle que l'on ne peut plus qualifier de synthétique tant elle se déploie en une multiplicité d'autres règles et ce, parce que l'on cherche, à travers elle, à répondre à des situations diverses parce que son application devra obéir à des modalités particulières; une règle qui semble relever du discours managérial plus encore que de la norme juridique.

On en veut pour preuve deux exemples de règles récemment créées par le Conseil d'État. Ainsi, l'arrêt Arcelor de 2007, dans lequel le Conseil d'État tire les conséquences, pour le juge administratif, de l'existence d'une obligation constitutionnelle de transposition des directives européennes :

"Considérant que si, aux termes de l'article 55 de la Constitution, "les traités ou accords régulièrement ratifiés ou approuvés ont, dès leur publication, une autorité supérieure à celle des lois, sous réserve, pour chaque accord ou traité, de son application par l'autre partie ", la suprématie ainsi conférée aux engagements internationaux ne saurait s'imposer, dans l'ordre interne, aux principes et dispositions à valeur constitutionnelle ; qu'eu égard aux dispositions de l'article 88-1 de la Constitution, selon lesquelles "la République participe aux Communautés européennes et à l'Union européenne, constituées d'États qui ont choisi librement, en vertu des traités qui les ont instituées, d'exercer en commun certaines de leurs compétences", dont découle une obligation constitutionnelle de transposition des directives, le contrôle de constitutionnalité des actes réglementaires assurant directement cette transposition est appelé à s'exercer selon des modalités particulières dans le cas où sont transposées des dispositions précises et inconditionnelles; qu'alors, si le contrôle des règles de compétence et de procédure ne se trouve pas affecté, il appartient au juge administratif, saisi d'un moyen tiré de la méconnaissance d'une disposition ou d'un principe de valeur constitutionnelle, de rechercher s'il existe une règle ou un principe général du droit communautaire qui, eu égard à sa nature et à sa portée, tel qu'il est interprété en l'état actuel de la jurisprudence du juge communautaire, garantit par son application l'effectivité du respect de la disposition ou du principe constitutionnel invoqué ; que, dans l'affirmative, il y a lieu pour le juge administratif, afin de s'assurer de la constitutionnalité du décret, de rechercher si la directive que ce décret transpose est conforme à cette règle ou à ce principe général du droit communautaire ; qu'il lui revient, en l'absence de difficulté sérieuse, d'écarter le moyen invoqué, ou, dans le cas contraire, de saisir la Cour de justice des Communautés européennes d'une question préjudicielle, dans les conditions prévues par l'article 234 du Traité instituant la Communauté européenne ; qu'en revanche, s'il n'existe pas de règle ou de principe général du droit communautaire garantissant l'effectivité du respect de la disposition ou du

\footnotetext{
14 Maryse Deguergue, «Les obiter dicta dans la jurisprudence du Conseil d'État », in Philippe RAIMBAULT (dir.), La pédagogie au service du droit, LGDJ, 2011, p. 229.

${ }^{15}$ Bruno LASSERRE, « Jugements », Juris-Classeur Administratif, cité par LE BOT, 2010.
} 
principe constitutionnel invoqué, il revient au juge administratif d'examiner directement la constitutionnalité des dispositions réglementaires contestées $1{ }^{16}$.

Le lecteur parvenu jusque-là, pourra également apprécier cet autre extrait dans lequel le Conseil d'État décrit la nouvelle procédure, créée par lui de toutes pièces, au terme de laquelle tout tiers peut envisager de contester un contrat administratif :

"Considérant que, saisi ainsi par un tiers dans les conditions définies ci- dessus, de conclusions contestant la validité du contrat ou de certaines de ses clauses, il appartient au juge du contrat, après avoir vérifié que l'auteur du recours autre que le représentant de l'État dans le département ou qu'un membre de l'organe délibérant de la collectivité territoriale ou du groupement de collectivités territoriales concerné se prévaut d'un intérêt susceptible d'être lésé de façon suffisamment directe et certaine et que les irrégularités qu'il critique sont de celles qu'il peut utilement invoquer, lorsqu'il constate l'existence de vices entachant la validité du contrat, d'en apprécier l'importance et les conséquences ; qu'ainsi, il lui revient, après avoir pris en considération la nature de ces vices, soit de décider que la poursuite de l'exécution du contrat est possible, soit d'inviter les parties à prendre des mesures de régularisation dans un délai qu'il fixe, sauf à résilier ou résoudre le contrat ; qu'en présence d'irrégularités qui ne peuvent être couvertes par une mesure de régularisation et qui ne permettent pas la poursuite de l'exécution du contrat, il lui revient de prononcer, le cas échéant avec un effet différé, après avoir vérifié que sa décision ne portera pas une atteinte excessive à l'intérêt général, soit la résiliation du contrat, soit, si le contrat a un contenu illicite ou s'il se trouve affecté d'un vice de consentement ou de tout autre vice d'une particulière gravité que le juge doit ainsi relever d'office, l'annulation totale ou partielle de celui-ci ; qu'il peut enfin, s'il en est saisi, faire droit, y compris lorsqu'il invite les parties à prendre des mesures de régularisation, à des conclusions tendant à l'indemnisation du préjudice découlant de l'atteinte à des droits lésés " 17.

Lisible? Parlons-en. Au terme des longs développements qu'il consacre au style rédactionnel, le Groupe de travail préconise, principalement, l'abandon de la phrase unique et des «considérant» pour les remplacer par des phrases courtes, ponctuées de points ainsi qu'une présentation en paragraphes courts afin de garantir « la rigueur du raisonnement » et de renforcer la « lisibilité de la décision ». La notion de « lisibilité » reste difficile à cerner : une décision n'est jamais lisible en soi mais seulement relativement à des lecteurs ${ }^{18}$. À lire le rapport Martin, il ressort que la syntaxe des juridictions administratives « rend plus difficile la lecture des décisions de justice par le public français » et qu'elle " en éloigne encore plus les lecteurs étrangers ${ }^{19}$. Le problème auquel se heurte le Conseil d'État est ainsi celui de la traduction de ses décisions, «qui comporte un risque d'erreur lorsque les subordonnées sont nombreuses ». L'enjeu est donc bien la faculté de la juridiction

\footnotetext{
${ }^{16}$ CE Ass., 8 février 2007, Société Arcelor Atlantique et Lorraine (req. 287110).

${ }^{17}$ CE, Ass., 4 avril 2014, Département du Tarn et Garonne (req. 358994).

C'est au demeurant une question lancinante qui n'a jamais trouvé de réponse satisfaisante, $v$. Bernard DuCAmin, "Les réactions d'un public cultivé », Etudes et Documents du Conseil d'État (36), Dossier « la langue française et le droit », 1984, p. 129-145.

${ }^{19}$ Rapport Martin, p. 11: «Ce style rend également, dans une certaine mesure, plus difficile leur diffusion à l'étranger, préoccupation qui, si elle ne saurait être déterminante, n'est pas sans poids dans la perspective d'une intégration normative européenne à laquelle participent nécessairement les juridictions nationales $»$.
} 
administrative à faire connaître sa jurisprudence, à la transmettre et donc à la communiquer à d'autres (et non à communiquer "sur » elle, ce qui suppose un discours sur le discours -, celui des communiqués de presse - que le Conseil d'État pratique désormais volontiers) ${ }^{20}$.

En s'efforçant d'adopter une rédaction sinon plus claire ou du moins plus simple de ses décisions, le Conseil d'État se donnerait les moyens de prendre part à ce que ses membres aiment appeler le « dialogue des juges ». Ce dernier ne consiste pas seulement, on le sait, en un aimable échange de vues entre cours nationales et supranationales, mais aussi en une "quête d'influence " " à plusieurs strates et dans plusieurs directions : les juges nationaux cherchent préventivement à convaincre du bien-fondé de leurs décisions, mais aussi à peser autant que faire se peut sur les décisions des juges supranationaux en développant des instruments procéduraux parfois très subtils voire éminemment complexes ${ }^{22}$.

On mesure, combien l'objectif poursuivi par le rapport Martin - améliorer « l'intelligibilité des décisions de justice » et « permettre d'exprimer aussi clairement, précisément et complètement que possible les motifs pour lesquels le juge adopte une solution » - exige que le juge administratif adapte son style, c'est-à-dire, ici, son écriture. Tout comme on mesure, par l'ensemble des propositions faites, les doutes que ces changements susciteraient si on les présentait aux justiciables - d'ailleurs non consultés et cruellement absents du Groupe de travail ${ }^{23}$.

\footnotetext{
${ }^{20}$ Rapport Martin, p. 39 : «Ces difficultés nuisent certainement à la diffusion du droit public français à l'étranger et notamment dans la sphère européenne, où la quasi-totalité des juridictions ont adopté des styles de rédaction plus modernes, y compris celles qui recouraient, comme la France - et probablement sous son influence - au style de la phrase unique ».

${ }^{21}$ Sir Basil MARKESINIS, "Judicial Style and Judicial Reasoning in England and Germany », Cambridge Law Journal, 59 (2), 2000, p. 294.

${ }^{22}$ On se permet de renvoyer à Pierre BRUNET, «Pluralisme juridique et hiérarchie des normes », in $\mathrm{P}$. BRUNET et F. ARENA (eds.), Questions contemporaines de théorie analytique du droit, Madrid, Marcial Pons, 2011, p. 53-74.

${ }^{23}$ Comme l'avait justement relevé Frédéric ROLIN « La disparition du considérant ou Georges Perec au Palais Royal » (7 août 2013) (http://frederic-rolin.blogspirit.com/archive/2013/08/07/la-disparitiondu-considerant-ou-georges-perec-au-palais-roy.html consulté le 23 avril 2015) qui faisait remarquer, d'une part, que les personnes composant le groupe de travail étaient des magistrats et non pas le tout venant ni l'usager et, d'autre part, que la lisibilité pourrait ne pas être appréciée de la même façon selon que l'on pense la juridiction des tribunaux et celle des cours administratives d'appel. Autant d'éléments qui, selon moi, attestent qu'on cherche à être lu et compris moins par des usagers de la justice que par leurs conseils, les juristes spécialisés, français ou étrangers et notamment les traducteurs des cours supranationales et étrangères. Ajoutons que le groupe de travail ne comprenait cependant ni traducteur juridique ni non plus de spécialiste de jurilinguistique.
} 
L'autre enjeu pour le Conseil d'État est de parvenir à remettre en cause le style de sa rédaction sans modifier le style de sa motivation tant la phrase unique est identifiée à la forme syllogistique laquelle constitue encore l'idéal de la motivation.

\section{Le " Formal Style " des décisions ou le syllogisme comme idéal}

Le laconisme du Conseil d'État en matière de motivation est légendaire ${ }^{24}$. En 1867, Adolphe Chauveau peut écrire : « le Conseil d’État ne manque jamais de motiver ses décisions ; cependant il est rare que les motifs soient autre chose que l'énonciation d'un principe assez vague ou une simple affirmation par laquelle il pose en principe la solution qu'il a cru devoir adopter sur la question qui lui est soumise »" II a pourtant semblé évoluer. Ainsi, en 1924, Marc Noël croit déceler que le Conseil d'État motive plus longuement ses décisions «au fur et à mesure qu'il apparaît davantage comme un véritable juge » et qu'il est « de moins en moins mêlé à l'administration ${ }^{26}$. Soixante ans plus tard, André de Laubadère juge pourtant qu'« en tant que méthode de style, la concision ne semble pas avoir été abandonnée d'une manière qui constitue vraiment un changement notable ${ }^{27}$. Le constat est d'une banalité telle qu'on a presque fini par le considérer comme inhérent au droit administratif $^{28}$. Le rapport Martin relève d'ailleurs que «[C]ertaines des motivations des décisions de la juridiction administrative se résument encore à de simples affirmations, qui ne font pas ou pas suffisamment apparaître les raisons de

\footnotetext{
${ }^{24}$ II suffit, pour s'en rendre compte, de se reporter à quelques grandes décisions très connues dans lesquelles le Conseil d'État se contentait d'une formule sibylline, reprise d'un arrêt à l'autre. Ainsi, Conseil d'État, 13 décembre 1889, Cadot : " du refus du maire et du conseil municipal de Marseille de faire droit à la réclamation du sieur Cadot, il est né entre les parties un litige dont il appartient au Conseil d'État de connaître (...) "; Conseil d'État, 6 février 1903, Terrier: du refus du préfet d'admettre la réclamation dont il l'a saisi il est né entre les parties un litige dont il appartient au Conseil d'État de connaître (...) ; Conseil d'État, 4 mars 1910, Thérond: du refus du maire et du conseil municipal de faire droit à cette réclamation il est né entre les parties un litige dont le Conseil d'État, compétent comme il vient d'être dit, est valablement saisi (...).

${ }^{25}$ Adolphe CHAUVEAU, Code d'instruction administrative, ou Lois de la procédure administrative, Paris : Cosse, Marchal et Cie, $3^{\mathrm{e}}$ édition, 1867, p. 243.

${ }^{26}$ Marc NoËL, «Les motifs dans les décisions des juridictions administratives », Revue du droit public, 1924, p. 350, ici p. 363.

${ }^{27}$ André DE LAUBADĖRE, « Le Conseil d'État et l'incommunicabilité », Études et documents du Conseil d'État, (31), 1979-1980, p. 17, ici p. 19.

${ }^{28}$ v. Roger PERROT, "Cour de cassation et Conseil d'État à travers leurs fonctions de juges suprêmes ", in Association des magistrats et anciens magistrats de la Cour de cassation (dir.), Le tribunal et la Cour de cassation 1790-1990. Volume jubilaire, Paris, Litec, 1990, p. 145 et Marie-Claire PONTHOREAU, "Réflexions sur la motivation des décisions juridictionnelles en droit administratif français », Revue du droit public, 1994, p. 747.
} 
l'interprétation que le juge donne de la règle de droit et de son application aux faits de l'espèce ${ }^{29}$. On ne compte en effet plus le nombre d'arrêts qui, d'hier à aujourd'hui, s'ouvrent par l'affirmation péremptoire d'une règle générale dont personne ne connaissait l'existence avant que le Conseil d'État ne la pose et dont nul ne pourrait connaître l'origine par la seule lecture de la décision ${ }^{30}$.

On a trouvé à ce laconisme des justifications diverses : la marque de l'autorité du roi sous la Monarchie de Juillet $^{31}$; celle de la prudence sous la Ille République ${ }^{32}$; une volonté de conserver une marge de liberté un peu plus $\operatorname{tard}^{33}$ et, aujourd'hui, celle d'exercer une influence ${ }^{34}$. En réalité, le souci de l'autorité n'a jamais complètement disparu. Seule sa forme d'expression a changé : les membres du Conseil d'État reconnaissent eux-mêmes que la technique de "l'affirmation synthétique » présente l'avantage de placer la réponse du juge « sous le sceau de l'évidence » et de lui donner un « caractère solennel » ${ }^{35}$.

On peut envisager une explication plus « objective » qui s'attacherait à la situation institutionnelle du Conseil d'État lequel, dès l'origine, n'est pas une juridiction comme les autres. Ces dernières sont réputées se fonder sur des textes donnés car leur action est supposée déterminée par un code. Dépourvu de code, le Conseil d’État demeure quant à lui très largement le maître des prémisses de ses décisions. II

\footnotetext{
${ }^{29}$ Rapport Martin, p. 10.

${ }^{30}$ On pourrait multiplier les exemples bien au-delà des premiers arrêts du Conseil d'État.

31 Denis SERRIGNY, Traité de l'organisation de la compétence et de la procédure en matière contentieuse administrative dans leurs rapports avec le droit civil, 1, Paris : Joubert, 1842, Préface, p. IV : « Au Conseil d'État, c'est le roi qui parle, qui décide, qui ordonne : son langage doit être bref, concis, impératif. À la cour de cassation, ce sont des magistrats qui interprètent la loi, et qui doivent rendre raison aux justiciables de leurs décisions ". L'ouvrage est consultable en ligne à cette adresse : http://hdl.handle.net/2027/uc1.\$b577454. V. aussi Grégoire BIGOT, «Histoire de la motivation en droit public français » in S. CAUDAL (dir.), La motivation en droit public, Paris : Dalloz, 2013, p. 47 qui résume très justement: la science du Conseil d'État, à l'époque, « doit demeurer la science camérale des princes qui gouvernent » (ici p. 59).

32 Edouard LAFERRIĖRE, Traité de la juridiction administrative et des recours contentieux, $2^{\mathrm{e}}$ ed, Nancy : Berger-Levrault, 1896, tome I, p. 344 : « II y a eu, en effet, dans la jurisprudence du Conseil, une période assez longue pendant laquelle la sobriété des motifs, surtout sur certains points de droit, a pu paraître excessive. Elle s'inspirait d'un sentiment de réserve et de prudence qui n'était souvent que trop justifié par l'état d'une législation et d'une jurisprudence encore mal définies et dont il importait de ne pas enrayer prématurément les progrès par des formules non arrêtées "

33 Jean RIVERO, "Le Conseil d'État, cour régulatrice », Rec. Dalloz, 1954, p. 157, relevant que le Conseil d'État ne souhaite pas « se ligoter par des formules trop strictes ».

${ }^{34}$ Christophe FARDET, « Le style des décisions du Conseil d'État » art. cit, p. 229.

${ }^{35}$ Frédéric LENICA, Julien BOUCHER, "Hiérarchie des normes et contentieux de la responsabilité ", AJDA, 2007, p. 585 s. (à propos de l'arrêt d'Assemblée du Conseil d'État du 8 février 2007, Gardedieu).
} 
aurait alors pu se transformer en un juge à l'anglaise dont on dit parfois qu'il est à sa propre décision ce qu'est la doctrine académique française aux décisions civiles ${ }^{36}$. Mais il se trouve pris dans une forme de dilemme: d'un côté, pour ressembler à n'importe quel juge, il doit se soumettre à l'article 5 du code civil ${ }^{37}$ qui interdit les arrêts de règlement et véhicule l'idée que le juge doit se contenter d'obéir à la loi positive ; de l'autre, étant dépourvu de code, c'est à lui qu'il revient de fabriquer de toutes pièces les règles générales encadrant son action et celles de l'administration. En les tenant pour données une fois pour toutes, sans les justifier, le Conseil d'État évite de mettre en évidence toutes les appréciations subjectives dont dépend le choix de ses prémisses. Ajoutons un autre élément non négligeable : à la différence de la Cour de cassation, le Conseil d'État a toujours eu - a encore - besoin de justifier sa compétence, laquelle ne répond à aucun critère précis qui préexisterait au Conseil d'État lui-même. C'est donc à lui qu'il revient d'en formuler les limites ce qu'il ne peut faire qu'à l'aide de considérants généraux (sans visa) - pratique qui est à la fois proche et différente des attendus de principe de la Cour de cassation (lesquels doublent, parfois sans les citer, les règles du Code civil) ${ }^{38}$.

Reste que, comme d'autres cours non soumises à un code ou qui ne le sont que partiellement, le Conseil d'État pourrait justifier les prémisses majeures de ses décisions en s'appuyant sur des précédents ou en formulant des principes généraux, ou encore des lignes de conduite jurisprudentielle permettant sinon de prédire ses décisions futures, du moins d'orienter les justiciables. Or, il ressort très clairement du rapport Martin qu'une telle conception n'est pas à l'ordre du jour et que le formalisme - même tempéré - demeure.

Ainsi, d'une part, l'« idéologie orthodoxe » dont parlait Llewellyn fonctionne pleinement : le rapport n'envisage pas de remettre en cause la forme syllogistique du raisonnement juridique. Mieux, il identifie le raisonnement juridique « rigoureux » avec le syllogisme ${ }^{39}$. D'autre part, la motivation demeure entendue aussi strictement

\footnotetext{
${ }^{36}$ Jean-Louis GoUTAL, « Characteristics of Judicial Style in France, Britain and the U.S.A. », American Journal of Comparative Law, 24 (1), 1976, p. 43 ici p. 64.

37 « II est défendu aux juges de prononcer par voie de disposition générale et réglementaire sur les causes qui leur sont soumises ».

38 Je remercie Jean Louis Halpérin de m'avoir signalé ce point.

39 «Les qualités de rigueur et de précision des décisions de la juridiction administrative sont unanimement saluées. Elles tiennent essentiellement à l'application par le juge d'un syllogisme
} 
que possible. II y a, à cet égard, un contraste saisissant entre le diagnostic selon lequel nombre de décisions du Conseil d'État sont encore trop proches de la simple affirmation $^{40}$ et le remède envisagé : la motivation en droit doit s'en tenir au strict nécessaire ${ }^{41}$.

Ce faisant, le Groupe de travail réaffirme que la sécurité juridique est mieux garantie dès lors que les raisons des raisons, si l'on peut dire, sont tues. Cette opinion est depuis longtemps largement répandue en France ${ }^{42}$. Encore aujourd'hui, il n'est pas rare d'entendre des membres de la juridiction administrative dire que l'enrichissement de la motivation pourrait être utilisée «pour favoriser le développement de chicaneries contentieuses ${ }^{43}$. À l'inverse, on se souvient que Llewellyn voyait dans le Grand Style une meilleure garantie de la sécurité juridique des plaignants liée à une plus grande prévisibilité des décisions. L'argument qu'il développait nous concerne au premier plan: les décisions adoptées en «style formel », ou suivant un raisonnement purement déductif, et donc fondées sur les règles de droit positif, supposent des règles claires, or la pratique révèle que c'est impossible. Les juges qui veulent se borner à l'application des seules règles juridiques se trouvent dans la nécessité de choisir celles applicables mais ce choix résulte d'une décision dont les termes restent implicites et dissimulés aux plaignants. C'est pourtant là que réside le cœur de la décision car, une fois prise, n'importe quel

juridique rigoureux et à la clarté de son développement, qui ne retient que les éléments de fait et de droit qui déterminent nécessairement la solution du litige ». (Rapport Martin, p. 12)

40 "La concision, qui constitue l'une des caractéristiques des décisions des juridictions administratives, si elle est en général la traduction de la rigueur du raisonnement et du souci de n'exprimer que les raisons qui ont conduit à la solution donnée au litige, est parfois excessive » (Rapport Martin, p. 10).

${ }^{41}$ "De manière générale, ne doivent être mentionnées que les dispositions dont le juge fait application. La motivation de la décision ne doit contenir que ce qui justifie le dispositif, à l'exclusion de toute description générale de l'applicabilité des normes qui ne répondrait pas à une argumentation des parties ou à la nécessité de justifier de l'interprétation de la règle dont il est fait application » (Rapport Martin, p. 27).

${ }^{42}$ On ne peut pas ne pas ici penser à ce que disaient des observateurs de la Cour de cassation : Adolphe TOUFFAIT et André TUNC, "Pour une motivation plus explicite des décisions de justice notamment de celles de la Cour de cassation ", Revue trimestrielle de droit civil, LXXII (3), 1974, p. 487, ici p. 497 : le style des décisions judiciaires laisse place à un second danger. La Cour de cassation procède par voie d'affirmation. Le souci de la sécurité juridique (...) la conduit à reprendre les mêmes principes dans les mêmes termes, quelles que soient les discussions qu'ils ont pu susciter. Tout se passe donc comme si la Cour se considérait infaillible. On peut discuter la portée du dogme, non son existence. De là résulte un certain immobilisme, une constance excessive, une "force d'inertie" (Léon Husson) - certains ont dit : une certaine sclérose ». Ils concluaient : " le style actuel des décisions, notamment de la cour de cassation, c'est un peu la messe en latin » (p. 507).

${ }^{43}$ Didier RIBES, "La motivation des décisions du Conseil d'État ", in Fabrice HOURQUEBIE et MarieClaire PONTHOREAU (dir.), La motivation des décisions des cours suprêmes et des cours constitutionnelles, Bruxelles : Bruylant, 2012, p. 183, ici p. 185. 
argument technique peut être développé de façon logique et cohérente. Notons, enfin, pour éviter les clichés, que la brièveté, la concision et une recherche d'efficacité dans la motivation ne sont pas des spécificités françaises. Les juristes de common law les apprécient tout autant. Mais ils ne les identifient pas nécessairement au syllogisme ${ }^{44}$.

Le présupposé - fort classique - qui se dégage tant de la lecture du rapport Martin que de la jurisprudence française en général est que la motivation en droit doit se limiter à une pure déduction de la solution. Autrement dit, une décision juridictionnelle sera considérée comme motivée (et donc juridiquement justifiée) si et seulement si - elle est déduite ou inférée d'une règle générale elle-même mise en relation avec des faits décrits objectivement ${ }^{45}$. Inversement, la décision d'un juge ne serait pas justifiée si elle était fondée sur ses sentiments personnels, sur ses croyances subjectives ou sa seule volonté ${ }^{46}$.

Bien que très répandue, cette conception ne correspond pas à une description empirique des faits mais à un idéal que l'on impute en général à Beccaria ${ }^{47}$. Or, il est pour le moins trompeur de prendre l'idéal pour la réalité. Par ailleurs, quand bien même on prescrirait au juge de se limiter à une motivation strictement suffisante et

\footnotetext{
${ }^{44}$ Griffin B. BELL, «Style in Judicial Writing », Journal of Public Law, 15 (2), 1966, p. 214, ici p. 218 : "The careful writer - the stylist - has the desire and the capacity to gear the facts and the law-indeed the whole opinion-to the issue or issues presented. His self-discipline is sufficient unto the day. $\mathrm{He}$ seeks not to display all of his legal knowledge, but is satisfied for posterity to judge his efforts case by case. Here is the place for the perfectionist. $\mathrm{He}$ is the polisher who goes the last mile to insure the accomplishment of his goal : brevity, clarity, and precedential preciseness ॥. v. aussi J. WETTER, The Styles of Appellate Judicial Opinions, op. cit., p. 313 qui qualifiait le style canadien d'exercice de « legal barbarism » (« Its fondamental faults are: intellectual confusion, lack of discipline in literary composition, as weil as length and thoroughness exceeding the requirements of each case »).

${ }^{45}$ Jean-Louis GoUTAL, "Characteristics of Judicial Style in France, Britain and the U.S.A. ", art.cit., ici p. 45 : «French courts, with amazing regularity, ever since the Revolution, have practiced deduction and nothing but deduction »; et p. 60: "Here is where the reasoning pattern fits in: deduction, nothing but deduction; since deductive reasoning appears to secure certainty in the conclusion, it secures legitimacy in the order ». Cette idée de permanence depuis la Révolution vient de John P. Dawson, The Oracles of the Law, New York: Gryphon, 1968, p. 410-411 qui parle des juges français comme des " ventriloques experts".

${ }^{46}$ Autrement dit : Jules César peut répondre à Caius qui la lui demande que la raison de sa décision est « dans sa volonté "; les caprices du juge, eux, ne sont pas des raisons susceptibles de justifier sa décision.

${ }^{47}$ Cesare Beccaria, Des délits et des peines, traduction M. Chaillou de Lisy, Paris, s.n., 1773, chap. IV : « Dans toute affaire criminelle, le juge doit partir d'après un syllogisme parfait, dont la majeure est la loi générale, la mineure l'action conforme ou non à cette loi, et la conséquence l'élargissement ou la punition de l'accusé. Un raisonnement de plus, soit que le juge le fasse de son gré ou qu'il y soit forcé, ouvre la porte à l'incertitude et à l'obscurité ».
} 
efficace, on ne peut écarter une autre dimension du problème à savoir le choix de la norme générale et abstraite qui doit justifier la solution particulière et concrète.

Pour comprendre cette difficulté, il convient d'introduire une distinction entre deux niveaux de rationalité ou de justification des juges: la justification interne et la justification externe ${ }^{48}$.

La justification interne est le raisonnement déductif - la plupart du temps syllogistique - par lequel les juges justifient leur décision. Mais le syllogisme judiciaire, souvent présenté comme la simple subsomption de faits particuliers sous une règle générale susceptible d'aboutir en définitive à une décision particulière, est beaucoup plus complexe. II implique lui-même quatre opérations différentes : le choix d'une norme juridique valide susceptible de constituer la prémisse majeure; l'identification des faits pertinents; la subsomption des faits concrets sous la qualification abstraite prévue par la norme choisie; le choix des conséquences qui, parmi celles indiquées par la norme générale, seront finalement appliquées à la situation concrète $^{49}$. La complexité ne cesse d'ailleurs pas car chacune de ces étapes peut elle-même être décomposée en plusieurs autres ${ }^{50}$.

Si la justification interne exige donc que la conclusion soit cohérente avec les prémisses, la vérification de cette cohérence reste une affaire assez triviale et, comme on l'a noté, peut toujours faire l'objet d'une reconstruction ex post ${ }^{51}$. Mais si on s'interroge sur les raisons pour lesquelles les prémisses ont été choisies et mériteraient d'être acceptées, si donc on cherche à justifier les prémisses de la justification interne (ou du syllogisme), on entre dans la sphère de la justification

\footnotetext{
48 Jerzy WRÓBLEWSKI, « Legal Decision and Its Justification », Logique et Analyse, 14 (53-54), 1971, p. 403 not. p. 413 et « Paradigms of Justifying Legal Decisions », in Aleksander PECZENIK, Lars LINDAHL and Bert VAN Roermund (eds.), Theory of Legal Science. Proceedings of the Conference on Legal Theory and Philosophy of Science, (Lund, Sweden, Dec. 11-14, 1983), Dordrecht: Reidel, $1984, p$. 253 : "Justification is thought of as giving arguments supporting a judicial decision. (...) The decision is internally rational when it is based on the premisses it accepts. The premisses themselves, however, can be also analyzed and either approved of or rejected, and depending on that the decision is treated as externally rational or not. " .

49 Jerzy WRÓBLEWSKI, « II modello teorico dell'applicazione della legge », Rivista Internazionale di Filosofia del Diritto, XLIV (1), 1967, p. 10, not. 10-21.

${ }^{50}$ Paolo Comanduccl, « II ragionamento giudiziale : lineamenti di un modello », in Mario BESSONE (ed.), Interpretazione e diritto giudiziale. I. Regole, metodi, modelli, Torino : Giappichelli, 1999, p. 4788, not. p. 55.

51 Aulis AARNIO, The Rational as Reasonable. A Treatise on Legal Justification, Dordrecht: Reidel, 1986, p. 120.
} 
externe. Cette justification joue un rôle considérable ${ }^{52}$ mais elle consiste en raisonnements le plus souvent persuasifs et non déductifs ${ }^{53}$ et ne peut pas être expliquée à l'aide d'instruments logico-formels ${ }^{54}$.

On comprend alors que les juridictions qui adhèrent avec force à l'idéal syllogistique cherchent à masquer ou du moins à ne pas inclure cet aspect de la justification de leurs décisions. Il est d'ailleurs frappant de constater que la très grande différence de style de la motivation tient bien souvent à cette justification externe, plus encore qu'à la brièveté ou la longueur des décisions juridictionnelles.

Or, au sein du Conseil d'État, c'est précisément à cette même justification externe que sont consacrées les conclusions des rapporteurs publics.

\section{Le " Grand Style » mezza-voce des conclusions}

Par le biais des conclusions des rapporteurs publics, lesquelles sont largement diffusées et facilement accessibles, la juridiction administrative accorde une grande place à des arguments autres que ceux tirés de la seule application du droit. La doctrine leur reconnaît un rôle capital ${ }^{55}$ et que d'aucuns contestent ${ }^{56}$. Quoi qu'il en

\footnotetext{
52 Aulis AARNIO, ibid: «the central problems of legal justification are connected with the external justification. (...) the difficulty that divides interpreters into different camps is tied, on one hand, to the choice and content of the premises, and, on the other hand, to the way in which the suitable principles of inference or basic values are chosen. The problem of legal discourse is thus concentrated upon the external justification ».

${ }^{53}$ Aulis AARNIO, op. cit., p. 122: "The external justification is not syllogistic at all. It is a matter of convincing the other part, the addressee of the interpretation. This is done simply so that the interpreter adds new syllogisms supporting his interpretation or his arguments, but every step is guided only in a "loose" way by the standards of rationality or by the standards of legal interpretation. There are no exact criteria on the basis of which one could say that just this argument (syllogism) is the proper one. The interpreter will succeed if the totality of arguments (the chain of syllogisms) is convincing enough for the other part ; v. aussi Riccardo GUASTINI, Interpretare e argomentare, Milano : Giuffrè, 2011, p. 258.

${ }^{54}$ Jerzy WRÓBLEWSKI, « II sillogismo giuridico e la razionalità della decisione giudiziale " (1974) in P. COMANDUCCI e R. GUASTINI (ed.), L'analisi del ragionamento giuridico. Materiali ad uso degli studenti, I, Torino : Giappichelli, 1987, 277-298.

55 André DE LAUBADĖRE, art cit.: " c'est en lisant les conclusions que l'on comprend que le juge consacre un nouveau critère (...) » (p. 18).

${ }^{56}$ Bruno Genevols, «Le commissaire du gouvernement devant le Conseil d'État statuant au contentieux ou la stratégie de la persuasion », RFDA 2000, p. 1207 : la doctrine a[-t-elle] tendance à surestimer le rôle joué par les commissaires du gouvernement. En la matière, nous sommes, pour notre part, enclin à adhérer au jugement mesuré que portait le président Odent: «tantôt le commissaire du gouvernement exerce une influence personnelle considérable sur la formation de la jurisprudence ; tantôt il se borne à être le porte-parole d'une sous-section : cela dépend de l'espèce et des tempéraments en présence » (cf. R. Odent, Contentieux administratif, 1977-1980, p. 1226).
} 
soit, elles occupent une place essentielle dans certaines revues juridiques qui leur ouvrent largement leurs pages.

Toutefois, pas plus que les rapports des avocats généraux, les conclusions des rapporteurs publics ne peuvent être formellement considérées comme faisant partie de la décision elle-même. Aucun juriste français ne songerait à les associer à la décision au moment de gloser sur le thème général de la motivation des décisions du Conseil d'État. Mieux encore, on peut aisément penser qu'elles contiennent d'autant plus d'éléments de politique jurisprudentielle que leurs auteurs se savent libres à l'égard de la formation de jugement. Leur contenu changerait beaucoup si elles devaient être considérées comme parties intégrantes de la décision. Et on peut aisément imaginer que les membres du Conseil d'État s'opposeraient fermement à ce qui apparaîtrait comme une rupture radicale avec une solide tradition.

Est-ce l'influence des travaux de Mitchel Lasser ? Le fait est que le rapport Martin présente les conclusions sous un jour assez nouveau ${ }^{57}$. II rappelle certes que des raisons d'ordre "pratique, procédural et juridique » font obstacle à ce que les conclusions «soient regardées comme participant à la motivation des décisions juridictionnelles »: elles n'engagent que le rapporteur, n'ont pas à être écrites et peuvent le cas échéant n'être connues que des personnes qui assistent à la séance publique; enfin, elles n'ont aucune autorité de choses jugée laquelle s'attache uniquement à la décision ; c'est donc elle qui doit être motivée. Et donc « le groupe de travail est fermement d'avis que les conclusions ne sauraient pallier les éventuelles insuffisances de motivation des décisions juridictionnelles ${ }^{58}$. Notons en passant que la concession n'est pas négligeable. Mais le rapport souligne aussi l'importance des conclusions « dans l'élaboration de la jurisprudence et dans la compréhension par les justiciables et par le public de ses évolutions » et les voit comme «la partie visible des "travaux préparatoires" de la décision de justice » qui permettent « aux parties de vérifier que leur affaire a été correctement traitée, en fait comme en droit »; de même, le rapport relève qu'elles «contiennent (...) des informations importantes pour la bonne compréhension de la décision qui n'ont pas leur place dans ses motifs : les solutions alternatives non retenues ; la portée de la solution retenue au regard de l'état du droit; les justifications d'une évolution de

\footnotetext{
${ }^{57}$ Les citations qui suivent sont aux pages 12 et 13 du rapport.

${ }^{58}$ Rapport Martin, p. 13.
} 
jurisprudence... ». Le rapport en recommande donc une diffusion plus large et plus aisée.

II convient d'insister sur la fonction des conclusions : c'est un euphémisme de dire qu'elles contiennent « des informations importantes » et il est douteux que ces informations soient considérées comme n'ayant pas leur place dans la motivation. Ou plutôt, considérer que le contenu des conclusions ne peut faire office de motivation de la décision, c'est déjà présupposer un certain concept de « motivation " - une motivation réduite à la justification purement interne de la décision - et donc un certain style.

Cela ne signifie pas nécessairement que les conclusions mettent en lumière des arguments "non juridiques» - politiques, idéologiques... - que les juges déguiseraient par la suite en arguments juridiques. On serait presque tenté de dire que c'est le contraire : les rapporteurs publics veillent bien souvent à mettre en avant les arguments susceptibles de contraindre juridiquement les juges à adopter la solution de principe qu'ils leur proposent. Les conclusions prononcées au sein de la juridiction administrative font donc rarement la part belle à des arguments ouvertement « politiques » ou subjectifs. Cela peut s'expliquer. On ne convainc pas des juges attachés au formalisme en leur parlant le langage de la « libre recherche ». Par ailleurs, si le rapporteur s'exprime en son propre nom et n'est donc pas un juge, il a vocation à le devenir ; mieux vaut donc montrer pour lui qu'il « pense comme un juge ${ }^{59}$. C'est la raison pour laquelle les meilleurs arguments - et ceux majoritairement mobilisés - sont tirés : a) des grands principes - au rang desquels on trouve (selon les contentieux) : la séparation des pouvoirs, la prééminence de la Constitution (ou encore « la hiérarchie des normes »), la sécurité juridique - ; b) du respect de la répartition des compétences entre les ordres juridictionnels et selon les critères dégagés par la jurisprudence auxquels il arrive que la loi se soit ralliée ; c) de la compatibilité du droit français avec le droit européen: cet argument est évidemment le plus récent et sa fréquence la conséquence de la décision Nicolo de 1989 par laquelle le Conseil d'État a abandonné une jurisprudence traditionnelle

\footnotetext{
${ }^{59}$ Cf. Hein KöTZ, art. cit., p. 42 : « Never would a German judge dare to say - as an English judge did - that "it sometimes helps to assess the merits of a decision if one starts by noticing its results, and only after doing that allots to it the legal principles upon which it is said to depend". Of course, we all know that this is exactly what happens in the minds of German or French judges, but it would be highly improper to say so in a judgment, and a young judge would probably ruin his career if he were inclined to express his thoughts in this way $»$.
} 
faisant prévaloir la loi nationale postérieure et contraire sur les traités. II convient enfin d'ajouter que la quête d'influence qui vaut pour les juges, on l'a vu, vaut aussi pour les rapporteurs publics dont les destinataires ne sont plus les seules juridictions nationales ${ }^{60}$.

Si elles ne font donc pas ouvertement appel à des arguments politiques, les conclusions contiennent nombre d'éléments qui relèvent d'un discours « doctrinal » et illustrent une « manière de faire » typique du juge administratif - et notamment du Conseil d'État - qui tient en un partage des rôles fort différent de celui auquel pensait Llewellyn : à la décision, une justification interne aussi formelle et synthétique que possible, aux conclusions une justification externe plus articulée mais faisant néanmoins (et le plus possible) appel à des arguments de droit positif. Bien évidemment, le fond du propos dépend très largement de la question de droit ${ }^{61}$. Mais le contraste considérable entre la décision qui affirme plus qu'elle ne justifie et les conclusions qui justifient plus qu'elles n'affirment est une constante. II y a là une ligne de partage des mots, si l'on peut dire, que l'on ne franchit pas facilement.

Ainsi, un arrêt de 1995 nous apprit, sans autre forme d'explication et sans qu'aucun texte ne soit jamais cité à l'appui de cette affirmation, que « le respect de la dignité de la personne humaine est une des composantes de l'ordre public ${ }^{62}$. Les raisons de cette innovation ne se trouvaient que dans les conclusions. En l'espèce pourtant, compte tenu de l'état du droit positif et de la question posée, il fallut au commissaire du gouvernement invoquer certains arguments situés bien loin des textes ou des grands principes identifiés comme «juridiques » pour démontrer qu'à côté des notions classiques de salubrité, sécurité, tranquillité l'ordre public devait accueillir le respect de la dignité humaine. Ainsi, concluait-il : "Sans aller jusqu'à évoquer ici les Untermenschen ou "sous-hommes" stigmatisés comme tels par l'Allemagne nazie encore qu'il ne soit pas inutile de rappeler que les nains en faisaient précisément

\footnotetext{
${ }^{60}$ Bruno Genevols, «Le commissaire du gouvernement devant le Conseil d'État statuant au contentieux ou la stratégie de la persuasion », RFDA 2000 p. 1207 s. : "Tout en n'étant pas liés par la jurisprudence des autres juridictions, ni le commissaire du gouvernement, ni le Conseil d'État ne peuvent en faire abstraction. Dans ce contexte, le commissaire se trouve investi d'une mission nouvelle. II lui faut désormais convaincre un auditoire plus large que son auditoire habituel. Traditionnellement, le commissaire s'adresse à la formation de jugement, ainsi qu'aux avocats aux Conseils présents à la barre, aux représentants des administrations, au public et à la doctrine s'il envisage de publier ses conclusions. Dorénavant, le commissaire doit par-delà cet auditoire s'efforcer, si besoin est, de convaincre d'autres juges. "

${ }^{61}$ Pour bien faire, il faudrait multiplier les exemples. Et donc y consacrer un livre entier.

${ }^{62}$ Conseil d'État, Assemblée, 27 octobre 1995, Commune de Morsang-sur-Orge.
} 
partie -, on ne peut qu'être frappé par la parenté unissant un tel spectacle avec les jeux du cirque de l'Antiquité, dont on connaît le rôle de canalisation des pulsions sadiques de la population. On ne peut davantage s'empêcher de comparer le sort du nain ainsi exhibé avec celui réservé aux phénomènes de foire, tels que les frères siamois, victimes de régressions génétiques ou autres monstres en tous genres, qui tendent aujourd'hui fort heureusement à disparaître, mais dont un film comme Elephant Man, de David Lynch, a pu souligner, à juste titre, la situation de profonde détresse ${ }^{63}$.

La subjectivité n'est cependant pas non plus niée : souhaitant « faire reconnaître qu'il est d'intérêt général de permettre à tous les enfants, quelle que soit leur condition et leur origine sociale, d'accéder à cette activité culturelle coûteuse que constitue l'enseignement musical, en les faisant bénéficier au besoin de tarifs préférentiels ", un rapporteur public admet sans fard que « l'intérêt général est une notion d'une certaine plasticité, volontairement imprécise, qui permet au juge d'adapter les contours de sa jurisprudence aux aspirations ou aux nécessités de son temps ${ }^{64}$.

On pourrait multiplier les exemples de ce type d'interventions personnelles qui démentent le cliché d'un juge baignant dans les eaux froides du formalisme. Mais ces cas sont loin d'être aussi fréquents qu'on peut l'imaginer. Dès qu'ils le peuvent les rapporteurs publics mettent en avant, à l'appui des solutions qu'ils proposent, des arguments identifiés et identifiables par les juges comme aussi juridiques - et donc

\footnotetext{
${ }^{63}$ Patrick FRYDMAN, Conclusions sur CE, Ass., 27 octobre 1995, Commune de Morsang-sur-Orge, RFDA, 1995 p. $1204 \mathrm{~s}$. Et plus loin : « Au total et bien que l'absence, à ce jour, de toute jurisprudence tant administrative que judiciaire ayant précisément défini les contours de cette notion vous prive ici d'éléments de comparaison utiles, il nous apparaît que le spectacle critiqué doit bien être regardé comme portant atteinte a la dignité de la personne humaine. Aussi étendue que soit la liberté d'expression, une attraction consistant ainsi, en vue de flatter les plus vils instincts, à ravaler au rang d'objet une personne handicapée à raison même de son handicap, ne nous paraît en effet pas pouvoir trouver sa place dans une société civilisée. Les réactions du corps social précédemment évoquées, qui montrent à quel point ce spectacle a été ressenti comme une régression par rapport aux efforts d'intégration sociale des handicapés déployés dans les dernières décennies, ne font que renforcer cette analyse. Du reste, on pourrait être tenté de se demander si une attraction aussi méprisable que le lancer de nains ne porte pas, à la vérité, tout autant atteinte à la dignité des spectateurs qui y participent ou y assistent qu'à celle des nains eux-mêmes... ».

${ }^{64}$ Et il continue « À cet égard, vous n'ignorez pas les profondes fractures qui divisent aujourd'hui (...) la société française. (...) Dans ce contexte, admettre en 1997 qu'est d'intérêt général tout ce qui contribue à affermir ou rétablir le lien social ne nous paraît pas déraisonnable. Et nous croyons que la fréquentation des écoles de musique, pour marginale et anecdotique qu'elle puisse paraître, peut y prendre sa part ", Jacques-Henri STAHL, Conclusions sur CE, Section, 29 décembre 1997, Commune de Gennevilliers ; Commune de Nanterre, (2 espèces), RFDA, 1998 p. 539 s. sur ce point v. aussi Jacques CAILLOSSE, "L'argument sociologique dans l'administration », in Dominique FENOUILLET (dir.), L'argument sociologique : pluriel et singularité, Paris : Dalloz, 2015, p. 119.
} 
aussi politiquement neutres - que possible. Ils cherchent, en d'autre termes, à neutraliser la dimension politique des solutions proposées pour mieux en souligner la nécessité juridique. Ce qui ne veut pas dire que les solutions proposées, et celles éventuellement retenues, soient elles-mêmes neutres.

Un exemple parmi d'autres? En 2007, dans un litige opposant deux personnes privées - un chirurgien-dentiste et la Caisse autonome de retraite de cette profession - et dans lequel était mise en cause la conformité à la convention européenne des droits de l'homme d'une loi de validation d'un décret, le commissaire du gouvernement a refusé de se situer sur le terrain de la responsabilité pour faute de l'État alors qu'il reconnaissait, dans le même temps, d'une part, que la loi française avait méconnu la convention européenne et, d'autre part, que « si l'on devait se borner à faire application des catégories traditionnelles du droit de la responsabilité, c'est très certainement du régime de la responsabilité pour faute que devrait relever la responsabilité de l'État à raison des lois inconventionnelles ॥ ${ }^{65}$. II proposera donc que le Conseil d'État reconnaisse un cas suis generis de responsabilité de l'État pour méconnaissance de ses engagements internationaux mais... sans faute ${ }^{66}$.

Pour justifier cette solution, il fera discrètement appel à un argument tiré d'une éventuelle difficulté d'ordre politique que pourrait créer la reconnaissance d'un cas de responsabilité pour faute de l'État du fait de l'adoption d'une loi contraire à un traité en expliquant, d'une part, que « le constat d'une méconnaissance de la norme supérieure et la qualification de faute donnée à cette situation sont deux opérations juridiquement distinctes » et, d'autre part, « qu'aucune norme » n'habilite le Conseil d'État «à procéder à cette qualification à l'égard de la loi ». Puis il ajoute, subrepticement: " dès lors que sont en cause les pouvoirs respectifs de la justice administrative et du pouvoir législatif », le Conseil d'État ne peut procéder «à une telle opération juridique, aussi chargée de conséquences et de symbole, que sur la base d'une habilitation expresse » et conclut: «En l'espèce, nous n'en trouvons pas $"{ }^{67}$. D'autres arguments sont ensuite convoqués qui tendent tous à montrer que

\footnotetext{
${ }^{65}$ Luc DEREPAS, « La responsabilité du fait des lois en cas de méconnaissance des engagements internationaux », Conclusions sur CE, Ass., 8 février 2007, Gardedieu, RFDA 2007, p. 361 s.

${ }^{66}$ Fabrice Melleray me rappelle que l'interprétation de l'arrêt - et de son titrage - ont donné lieu à des discussions sans fin.

${ }^{67}$ Luc DEREPAS, ibid.
} 
le droit positif, national comme international et même comparé, n'obligent pas le juge qui constate une violation d'un traité par la loi, à qualifier cette violation de « faute ${ }^{68}$.

Ces deux exemples montrent combien le statut formel des conclusions contraste avec leur contenu mais aussi, dans le même temps, combien, ce contenu reste étroitement dépendant de la représentation que l'institution entend donner de son rôle et de la fonction de juger : dire le droit, rien que le droit, quand bien même ce juge se ferait le manager de sa propre décision.

Devant le style des jugements rédigés par Benjamin Cardozo, Richard Posner, luimême juge désormais, s'exclamait : "This is law as poetry " ${ }^{69}$. On peut parier qu'il faudra attendre encore longtemps avant que les arrêts ne parviennent à susciter la même réaction. On ne peut certes non plus exclure que les changements de forme envisagés aujourd'hui auront, à terme, des effets sur le fond des décisions, même s'il est à ce jour bien difficile d'en prédire la nature et la portée. Le changement substantiel qu'on pourrait espérer serait l'abandon de cette double croyance - double illusion - qu'il n'y a de rigueur de raisonnement que dans le syllogisme et de justification juridiquement légitime qu'interne. Et qui sait : peut-être que l'extension du contexte juridictionnel au sein duquel intervient le Conseil d'État aura un jour raison des habitudes les plus tenaces. Signe d'un changement, l'abandon de la phrase unique sera-t-elle le chant du cygne du formalisme ? II est permis d'en douter...

\footnotetext{
${ }^{68}$ Luc DEREPAS, ibid: "De ce tour d'horizon, nous tirons une conclusion et une conviction: la conclusion est que vos homologues étrangers ont le plus souvent estimé qu'ils n'étaient pas contraints par quelque norme impérative d'appliquer à la responsabilité du fait des lois inconventionnelles les règles de droit commun de la responsabilité administrative. La conviction est qu'ils ont fait preuve de prudence, en veillant à assurer la réparation des préjudices en cause tout en ne voulant pas paraître heurter les principes de la séparation des pouvoirs. Telle est pour notre part l'orientation que nous vous incitons à adopter $"$.

${ }^{69}$ Richard A. Posner, Cardozo : A Study in Reputation, Chicago : Univ. of Chicago Press, 1990, p. 102 , les formules en question étaient « Danger invites rescue. The cry of distress is the summons to relief » et " The risk of rescue, if only it be not wanton, is born of the occasion. The emergency begets the man ».
} 